\title{
Particle size and magnetic field-induced optical properties of magnetic fluid nanoparticles
}

\author{
G. Narsinga Rao, ${ }^{1,3}$ Y. D. Yao, ${ }^{1}$ Y. L. Chen, ${ }^{2}$ K. T. Wu, ${ }^{2}$ and J. W. Chen ${ }^{3}$ \\ ${ }^{1}$ Institute of Physics, Academia Sinica, Taipei, Taiwan, Republic of China \\ ${ }^{2}$ Department of Physics, Fu Jen University, Taipei, Taiwan, Republic of China \\ ${ }^{3}$ Department of Physics, National Taiwan University, Taipei, Taiwan, Republic of China
}

(Received 18 February 2005; revised manuscript received 15 June 2005; published 27 September 2005)

\begin{abstract}
Magnetite nanoparticles with diameters of 7, 9, and $12 \mathrm{~nm}$ have been prepared by a chemical coprecipitation method. The transmission of light through magnetic fluid containing these nanoparticles has been investigated as a function of film thickness with wavelength between 400 and $750 \mathrm{~nm}$, and applied magnetic fields up to 275 Oe. The transmission threshold shifts to the lower wavelength side with decreasing magnetic fluid film thickness as well as the particle size. For a given film thickness, the transmittance increases with increasing magnetic field for films with a particle size of 7 and $9 \mathrm{~nm}$, but decreases in the 12-nm film. This is attributed to the competition between the van der Waals and dipole-dipole interaction.
\end{abstract}

DOI: 10.1103/PhysRevE.72.031408

PACS number(s): 82.70.-y, 75.50.Mm, 78.20.Ls, 78.67.-n

\section{INTRODUCTION}

Nanoscale particles exhibiting a superparamagnetic behavior have been intensively studied recently due to the enhanced and novel applications in biomedical and diagnostic fields $[1,2]$. The optical, magnetic, chemical, and mechanical properties of the nanoparticles depend mostly on the particle shapes, size, composition, and external magnetic field. Recently, there has been considerable interest in magneto-optic devices which combine magnetic and optical phenomena $[3,4]$. The optical transmission of the magnetic fluid films under magnetic field is one of the important areas of magneto-optical effects. Some reports show that the optical transmission of the magnetic fluid films can be modulated by varying the magnetic field [5-7]. In addition to magnetic field effect, the temperature [8], and the wavelength [9] effect on the transmission of the magnetic fluid film were also reported. It is indicated that the optical transmission is attributed to the formation of the structural patterns of the magnetic fluid films under magnetic fields. On the other hand, Butter et al. [10] demonstrated for the first time the direct observation of dipolar chains in a ferrofluid in zero field using cryogenic electron microscopy. They found an abrupt transition from separate particles to randomly oriented linear aggregates with increasing particle size. However, the microstructures of a ferrofluid are expected to be influenced significantly by particle radius, particle concentration, and the thickness of their surfactant layer. We therefore synthesized magnetite nanoparticles with similar parameters (i.e., concentration and surfactant) with different particle sizes. In this paper, the effect of particle size on optical transmission of light through the $\mathrm{Fe}_{3} \mathrm{O}_{4}$ magnetic fluid has been investigated as a function of external magnetic field and incident optical wavelength with different film thickness. We found that the transmittance increases with increasing magnetic field for films with a particle size of 7 and $9 \mathrm{~nm}$, but decreases for the 12-nm films. The competition between van der Waals and dipole-dipole interactions is responsible for this significant transmittance variation with particle size.

\section{EXPERIMENTAL}

The synthesis of magnetite nanoparticles has been prepared by a chemical coprecipitation method. Distilled water was deoxygenated by bubbling with pure $\mathrm{N}_{2}$ gas for $1 \mathrm{~h}$ prior to the use. The solutions of ferric chloride hexahydrate $\left(\mathrm{FeCl}_{3} \cdot 6 \mathrm{H}_{2} \mathrm{O}\right)$ and ferrous chloride tetrahydrate $\left(\mathrm{FeCl}_{2} \cdot 4 \mathrm{H}_{2} \mathrm{O}\right)$ were prepared as a source of iron with molar ratio $2: 1$ by dissolving the respective chemicals in deoxygenated water under vigorous stirring. The solution of sodium hydroxide, $\mathrm{NaOH}(1 \mathrm{M}, 100 \mathrm{~mL})$, was slowly injected into the iron source under vigorous stirring in order to obtain a $\mathrm{pH}$ value of 8,9 , and 10 . This reaction was processing under the protection of $\mathrm{N}_{2}$ gas to avoid possible oxygen contamination during the process. The black precipitate was isolated in the magnetic field, and the supernatant was removed from the precipitate by decantation. After washing the precipitate three times, $0.01 \mathrm{M} \mathrm{HCl}$ was added to neutralize the anionic charge on the nanoparticle surface. The cationic colloidal

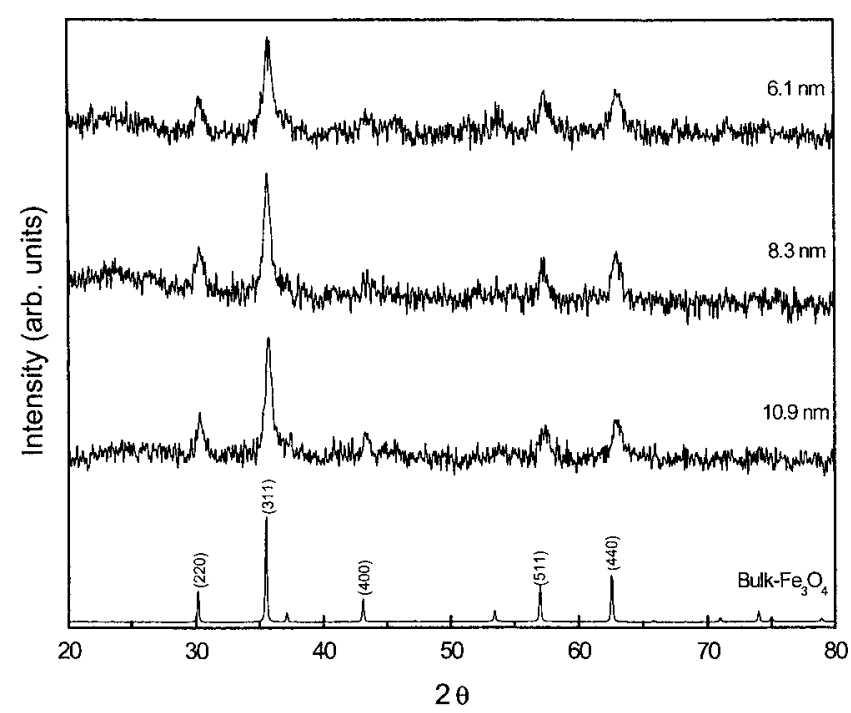

FIG. 1. X-ray-diffraction patterns $\mathrm{Fe}_{3} \mathrm{O}_{4}$ nanoparticles and bulk $\mathrm{Fe}_{3} \mathrm{O}_{4}$. 


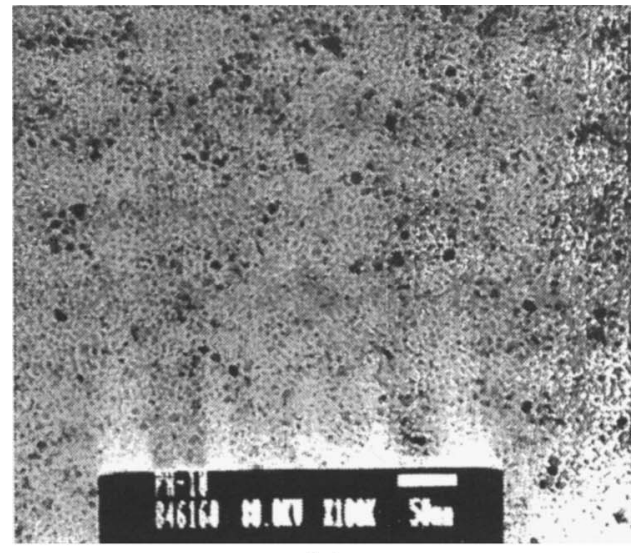

(a)

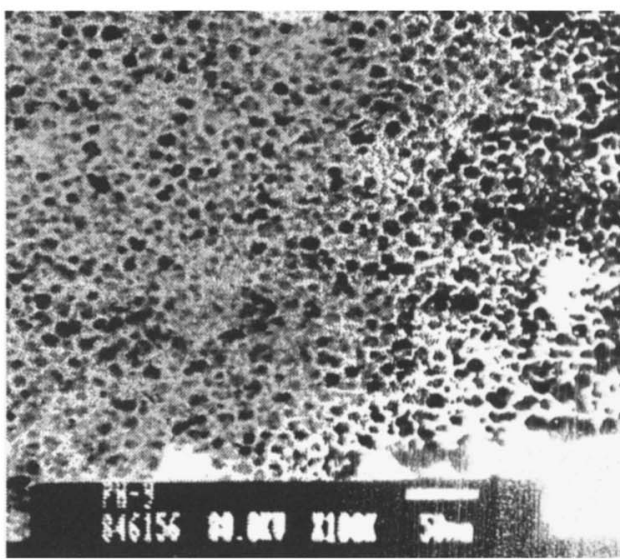

(b)

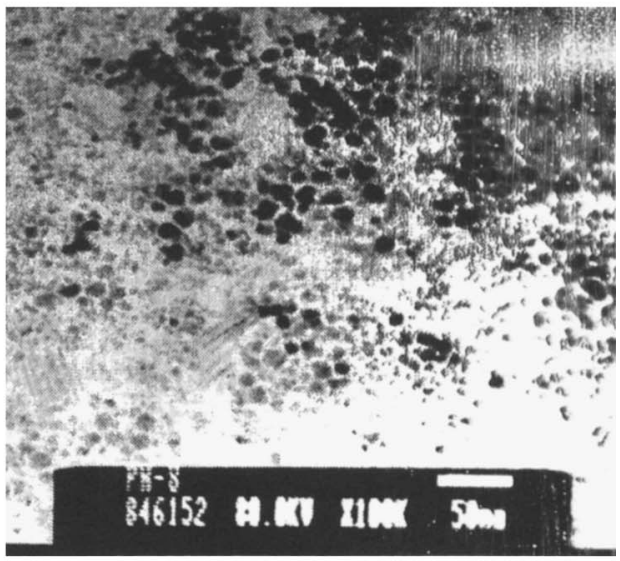

(c)

FIG. 2. Transmission electron micrographs of magnetic fluid nanoparticles prepared at (a) $\mathrm{pH} \mathrm{10,} \mathrm{(b)} \mathrm{pH} 9$, and (c) $\mathrm{pH}$ 8. The scale bar corresponds to $50 \mathrm{~nm}$.

particles were separated by centrifugation. Coating was carried out using surfactant (oleic acid) under vigorous stirring and dispersed in kerosene. The crystal structure and phase purity of the $\mathrm{Fe}_{3} \mathrm{O}_{4}$ nanoparticles were checked by powder $\mathrm{X}$-ray diffraction (XRD) using a Philips PW-1710 diffractometer with $\mathrm{Cu} K \alpha$ radiation at room temperature. The average particle size, shape, and morphology were examined using transmission electron microscopy (TEM). A drop of

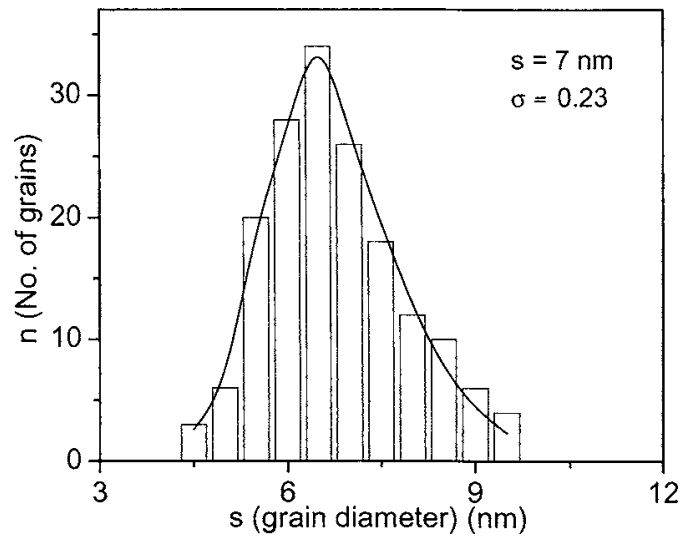

FIG. 3. Histogram of number of grains vs grain diameter for $7 \mathrm{~nm} \mathrm{Fe}_{3} \mathrm{O}_{4}$ particles. Solid curve represents the fitting of lognormal distribution function.

magnetic fluid is injected into glass cells with its depth varied between 10 and $1000 \mu \mathrm{m}$ to form a magnetic fluid film. The intensity of transmitted light can be measured as a function of incident wavelength between 400 and $750 \mathrm{~nm}$ and with applied magnetic fields up to 275 Oe for all the films. The magnetic fields were applied perpendicular to the magnetic fluid films.
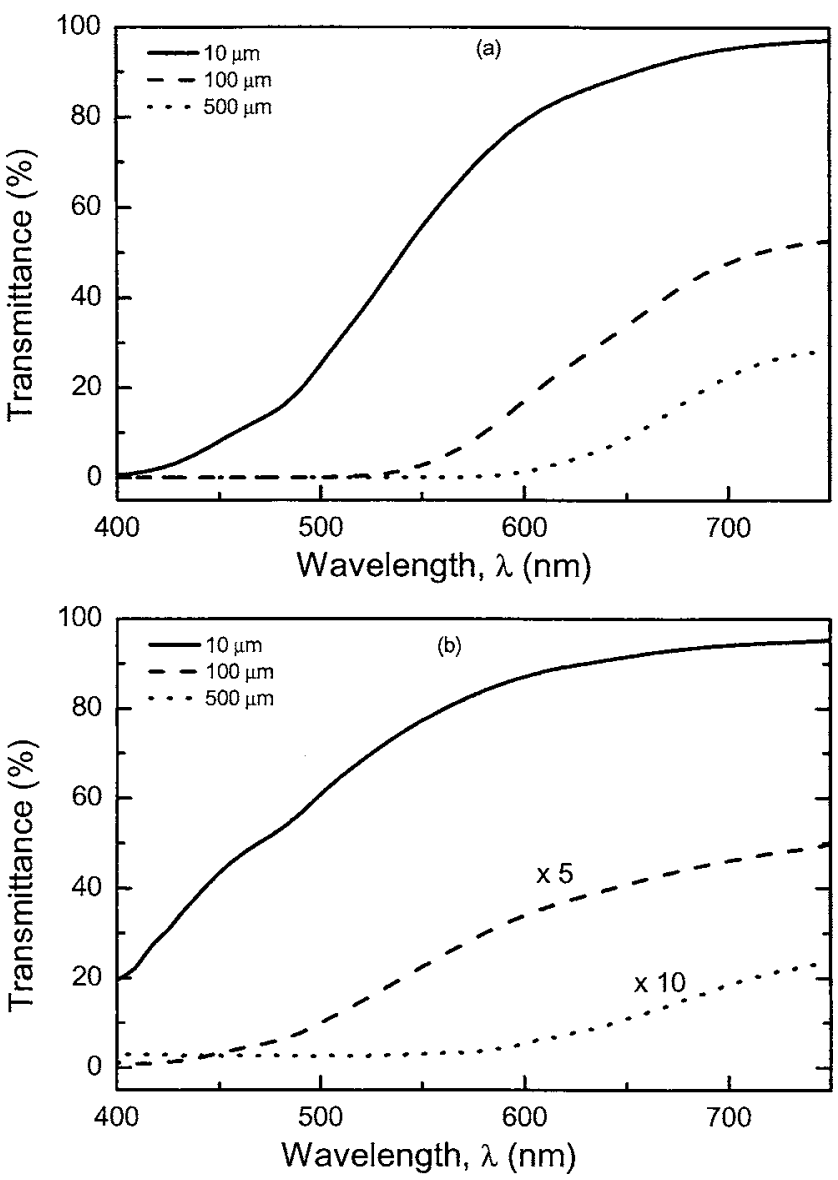

FIG. 4. The variation of the transmittance $(T)$ as a function of wavelength in zero magnetic field for particle size of (a) $12 \mathrm{~nm}$ and (b) $7 \mathrm{~nm}$ with different film thickness. 


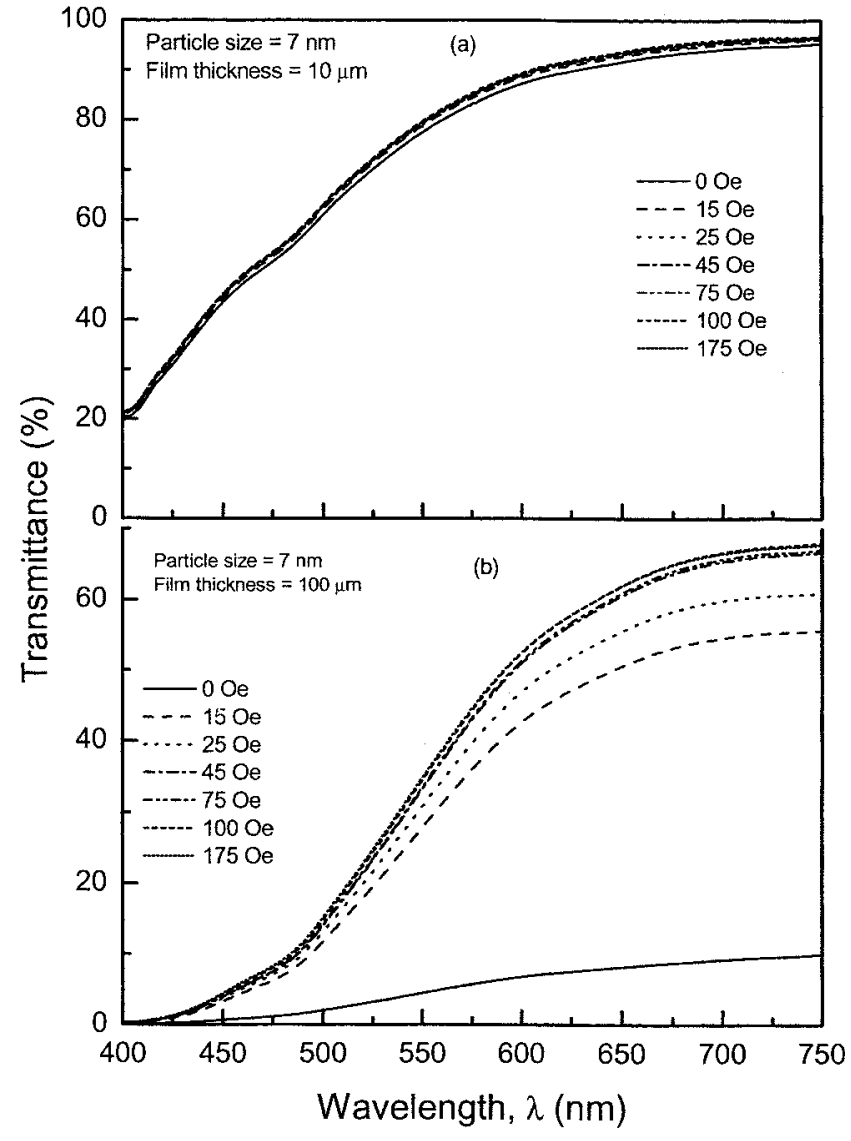

FIG. 5. The variation of the transmittance $(T)$ as a function of wavelength in magnetic field for particle size of $7 \mathrm{~nm}$ with film thickness of (a) $10 \mu \mathrm{m}$ and (b) $100 \mu \mathrm{m}$.

\section{RESULTS AND DISCUSSION}

The XRD patterns of $\mathrm{Fe}_{3} \mathrm{O}_{4}$ bulk and as-precipitated powder samples are shown in Fig. 1. The crystal structure of the nanoparticles is the same as that of the bulk $\mathrm{Fe}_{3} \mathrm{O}_{4}$. The average crystallite sizes of $\mathrm{Fe}_{3} \mathrm{O}_{4}$ prepared at a $\mathrm{pH}$ value of 8,9 , and 10 were found to be $10.9,8.3$, and $6.1 \mathrm{~nm}$, respectively, from the XRD peak width employing the Scherrer relation with instrumental correction. The TEM images of magnetic fluid nanoparticles prepared at (a) $\mathrm{pH} 10$, (b) $\mathrm{pH} 9$, and (c) $\mathrm{pH} 8$ are depicted in Fig. 2. The TEM images revealed a uniform spherical shape of the particles with different mean sizes. Figure 3 shows the typical particle size histogram obtained from the TEM data of the $\mathrm{pH} 10$ sample. The solid line represents the best curve fit (log-normal distribution [11]) of the particle size histogram. The mean diameters (geometric deviation) are found to be $7 \mathrm{~nm}(0.23)$, $9 \mathrm{~nm}(0.28)$, and $12 \mathrm{~nm}(0.32)$ for a $\mathrm{pH}$ value of 10,9 , and 8 , respectively. The particle diameters from TEM measurements are slightly larger than the observed crystal sizes from $\mathrm{XRD}$, due to the presence of noncrystalline surface layers.

Figure 4 shows the variation of the transmittance $(T)$ as a function of wavelength $(\lambda)$ in zero magnetic field for a particle size of (a) $12 \mathrm{~nm}$ and (b) $7 \mathrm{~nm}$ with different film thickness. In the case of $10-\mu \mathrm{m}$ films, the transmittance increases dramatically from zero to $97 \%$ with a particle size of $12 \mathrm{~nm}$

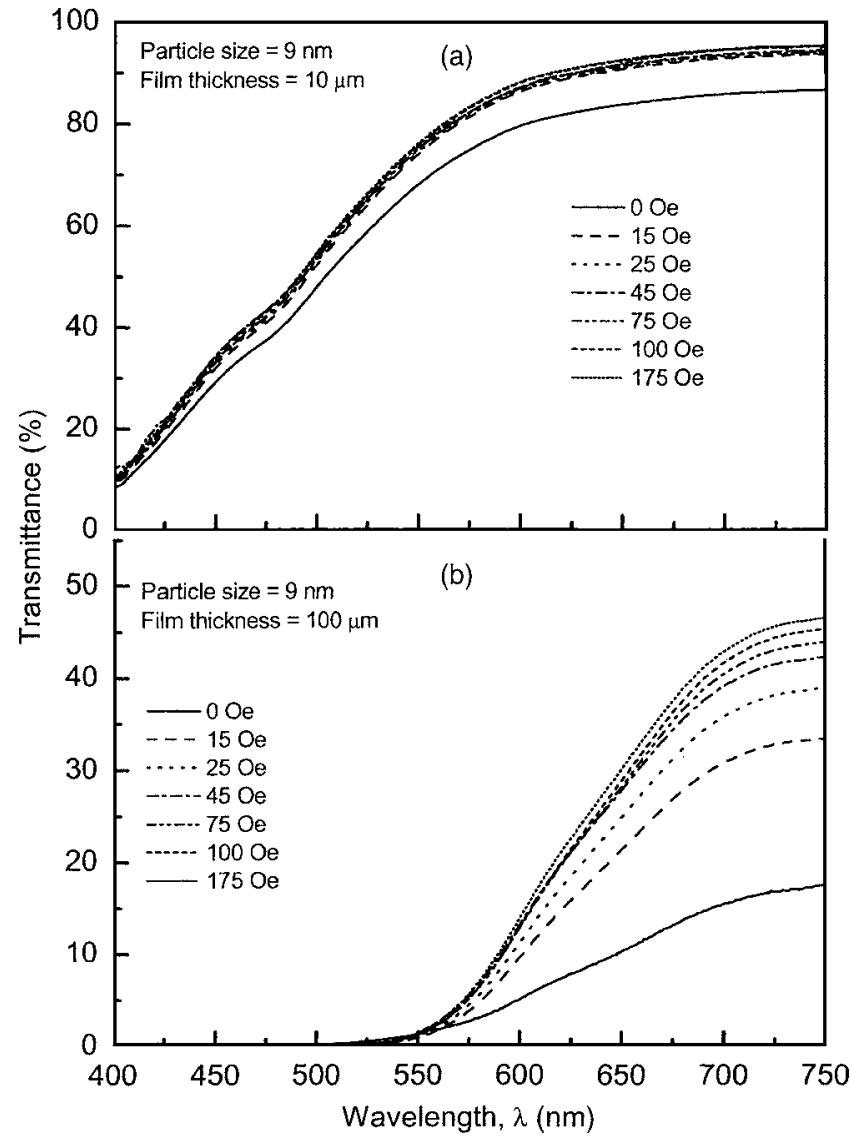

FIG. 6. The variation of the transmittance $(T)$ as a function of wavelength in magnetic field for particle size of $9 \mathrm{~nm}$ with film thickness of (a) $10 \mu \mathrm{m}$ and (b) $100 \mu \mathrm{m}$.

and from $20 \%$ to $95 \%$ with a particle size of $7 \mathrm{~nm}$, respectively, as the wavelength increases from 400 to $750 \mathrm{~nm}$. For films with a particle size of $12 \mathrm{~nm}$, the transmission threshold increases from 400 to $600 \mathrm{~nm}$ monotonically as the film thickness increases from 10 to $500 \mu \mathrm{m}$. The transmission thresholds are lower for films with a particle size of 7 and $9 \mathrm{~nm}$. This can be ascribed to the elongation of the traveling path as the light passes through the films [12]. Figures 5 and 6 depict the transmittance as a function of the wavelength in a magnetic field up to 275 Oe for (a) $10-\mu \mathrm{m}$ and (b) $100-\mu \mathrm{m}$ films with a particle size of 7 and $9 \mathrm{~nm}$, respectively. The variation of transmittance due to magnetic field is very small in the 10- $\mu \mathrm{m}$ film [Figs. 5(a) and 6(a)], whereas a dramatic increase of transmittance with magnetic field is observed in the 100- $\mu \mathrm{m}$ film [Figs. 5(b) and 6(b)] for both particles. Figure 7 shows the transmittance as a function of the wavelength in a magnetic field up to 275 Oe for (a) $10-\mu \mathrm{m}$ and (b) $100-\mu \mathrm{m}$ films with a particle size of $12 \mathrm{~nm}$. In the case of the $10-\mu \mathrm{m}$ film, the transmittance value increases with an increasing magnetic field below $\lambda=550 \mathrm{~nm}$, similar to that of films with 9 and $7 \mathrm{~nm}$ particles. Above $\lambda=600 \mathrm{~nm}$, the behavior is different: the transmittance value increases in low magnetic fields and decreases in higher magnetic fields. For the $100-\mu \mathrm{m}$ film [Fig. 7(b)], the transmittance value increases in lower magnetic fields $(>25$ Oe) $)$ but decreases with further increase of the magnetic field. For 


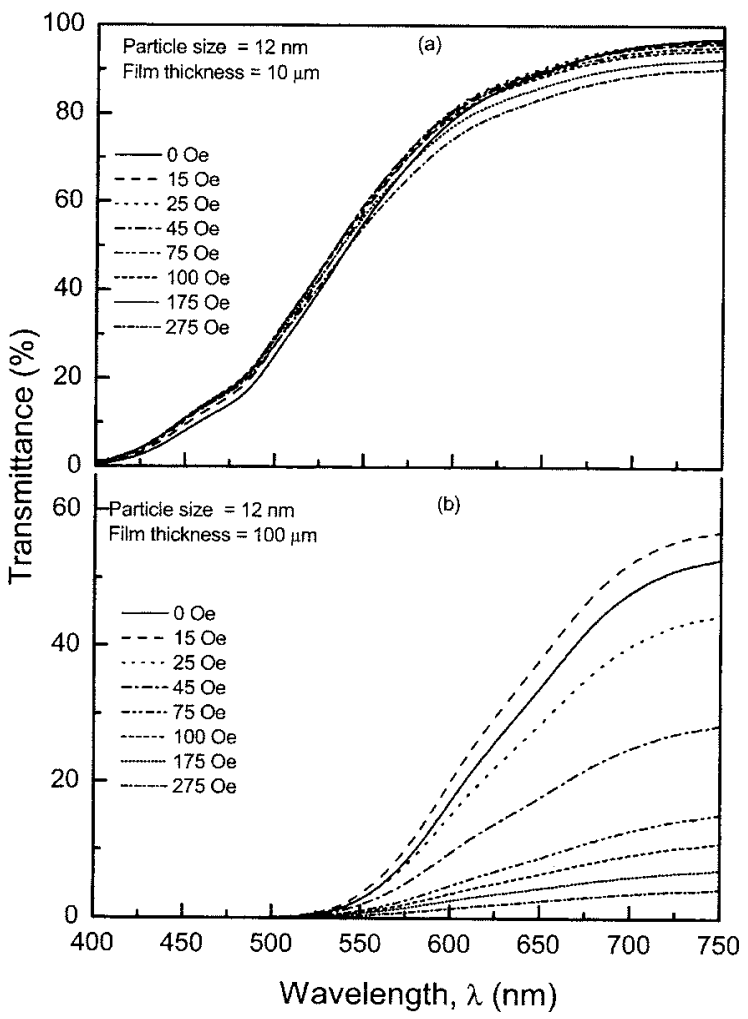

FIG. 7. The variation of the transmittance $(T)$ as a function of wavelength in magnetic field for particle size of $12 \mathrm{~nm}$ with film thickness of (a) $10 \mu \mathrm{m}$ and (b) $100 \mu \mathrm{m}$.

clearance, the magnetic field dependence of the transmittance value at $\lambda=750 \mathrm{~nm}$ for (a) $10-\mu \mathrm{m}$, (b) $100-\mu \mathrm{m}$, and (c) $500-\mu \mathrm{m}$ films with all particle sizes is depicted in Fig. 8. The sharp increase of transmittance value observed for all the films with a particle size of 7 and $9 \mathrm{~nm}$ at low fields is due to the homogeneous separation and spherical shape of the particles [Figs. 2(a) and 2(b)]. The variation of transmittance with the applied magnetic field is very small for the $10-\mu \mathrm{m}$ films [Fig. 8(a)] and is most pronounced for the $100-\mu \mathrm{m}$ films [Fig. 8(b)] for all samples. For a given film thickness, the transmittance increases with increasing magnetic field for films with a particle size of 7 and $9 \mathrm{~nm}$, but decreases in the 12-nm films.

The transmittance is an indicator of the structures formed in the fluid. Magnetic fluids exhibit a rich and highly varied phase and structural behaviors depending on the competition between van der Waals and dipole-dipole interactions of the colloidal particles. These interactions depend mainly on both the distance between the two particles and on their radii. The van der Waals interaction is isotropic and its strength of interaction is a function of $a_{\text {core }} / r_{d}$, where $a_{\text {core }}$ is a magnetic core radius and $r_{d}$ is the minimal interparticle distance (twice the sum of the core radius and the thickness of the surfactant layers), while the dipole-dipole interaction is anisotropic and is proportional to $a_{\text {core }}^{6} / r_{d}^{3}$ [13]. Thus, on increasing the particle size, the magnetic attraction between two dipoles will become more important. Recently, the magnetic attraction between two dipoles has been estimated and found to be dominant for oleic-acid-coated magnetic particle diameter

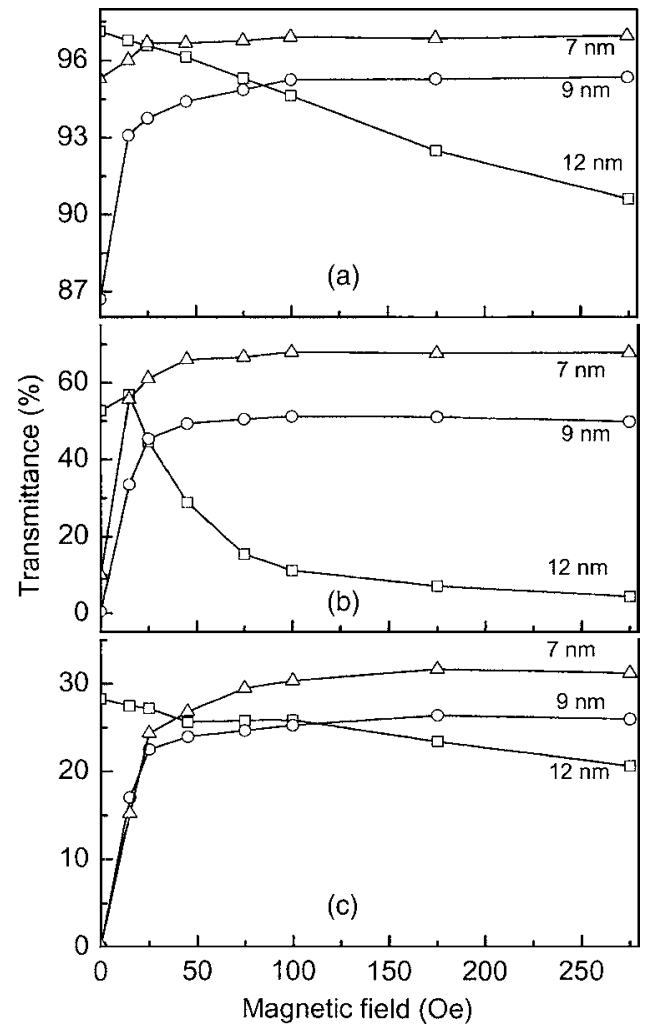

FIG. 8. The magnetic-field-dependent transmittance with incident wavelength of $750 \mathrm{~nm}$ for all samples with film thickness of (a) $10 \mu \mathrm{m}$, (b) $100 \mu \mathrm{m}$, and (c) $500 \mu \mathrm{m}$.

larger than $10 \mathrm{~nm}$ [13], while for smaller particles the van der Waals attraction is more important. The magnetic colloids used in our study consist of roughly spherical $\mathrm{Fe}_{3} \mathrm{O}_{4}$ particles, with a diameter of around 7, 9, and $12 \mathrm{~nm}$ (Fig. 2). All particles have oleic acid grafted onto their surfaces which makes them soluble in organic solvents. The thickness of the grafted oleic acid layer has not yet been conclusively determined, but a value of $1-2 \mathrm{~nm}$ is usually assumed. The magnetic fluid microstructures appear to be very sensitive to particle radius as well as the surfactant layer thickness. The variation of the transmittance is mainly dependent on the amount of light being scattered by the sample, which is determined by the size, shape, and number of scatterers in the magnetic fluid films under an applied magnetic field. To clarify the mechanism of the observed transmittance behaviors, we have studied their microscopic pictures. Figures 9 and 10 show the typical microscope photographs of the magnetic fluid under perpendicular magnetic fields for the $100-\mu \mathrm{m}$ films with a particle size of 7 and $12 \mathrm{~nm}$, respectively. At zero magnetic field, one can see the separated droplets randomly distributed in the films with a particle size of $7 \mathrm{~nm}$ [Fig. 9(a)] and for $9 \mathrm{~nm}$ particles (not shown here), while linear chains are observed in 12-nm films [Fig. 10(a)]. Similar experimental observations in ferrofluids at zero field have been reported recently by Butter et al. [10]. These microstructures suggest that the van der Waals force prevails in the films with 7- and 9-nm particles but the dipolar forces become strong enough to induce chain formation in 12-nm films $[13,14]$. Different microstructures were observed not 

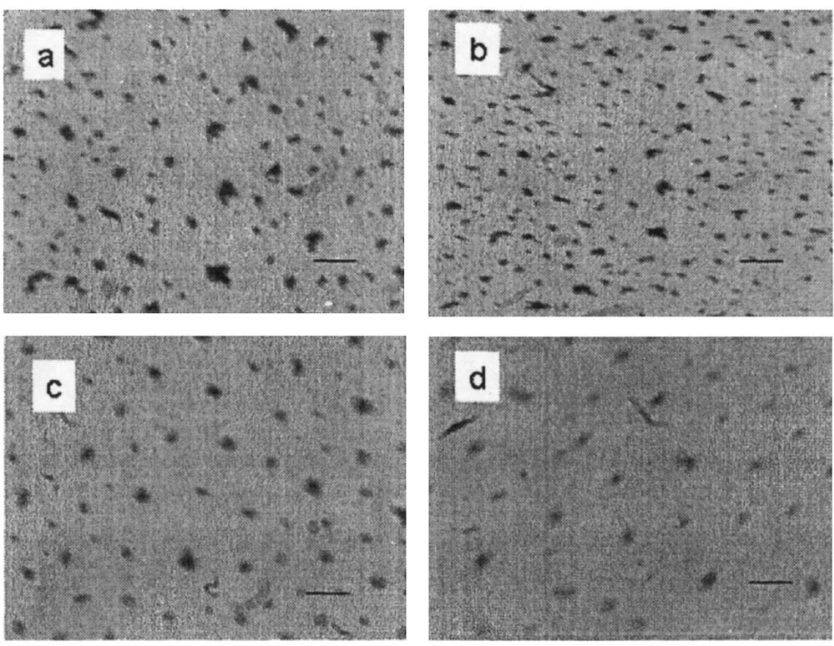

$\mathrm{OH}$

$\overline{40 \mu \mathrm{m}}$

FIG. 9. Optical micrographs of magnetic fluid with particle size of $7 \mathrm{~nm}$ at different magnetic fields perpendicular to the image plane (a) $H=0$, (b) $H=25 \mathrm{Oe}$, (c) $H=100 \mathrm{Oe}$, and (d) $H=210 \mathrm{Oe}$.

only in zero field but also in different applied magnetic fields. For the particle size of 7- and 9-nm films, the randomly distributed droplets start to form clusters with increasing magnetic field [Fig. 9(b)]. As a result, the number of scatterers and the cross section decrease, which causes an increase of the transmittance at low fields. The clusters become more tightly packed at higher fields [Figs. 9(c) and 9(d)] and hence the transmittance value is saturated. In contrast, the linear chain structures of the 12-nm films become orderly aligned clusters with increasing magnetic field [Figs. 10(b)-10(d)]. The strong dipole-dipole interactions in the 12-nm particle film are responsible for the particles approaching each other in the initial stage of formation of clusters. As a result, the area of the liquid space increases in low fields and it leads to the initial increase of transmittance. Under perpendicular magnetic field, the shape fluctuations in linear chains give rise to chain-chain interaction and the formation of thick sheets of particles (column) due to lateral (perpendicular to the chain axis) aggregation. When the magnetic field increases further, more columns are formed because the dipolar repulsive force in each column becomes dominant. The split of the column will cause the number of scatterers and the cross section to increase with increasing magnetic field and leads to a decrease of the transmittance. The size-dependent aggregation abilities due to the balancing between van der Waals and dipole-dipole interactions of magnetic fluid nanoparticles are responsible for this unusual
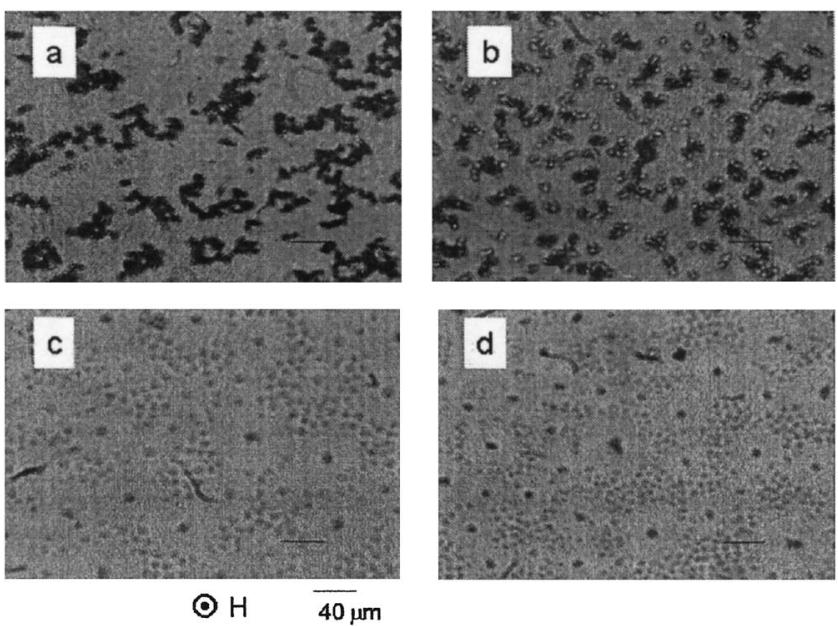

FIG. 10. Optical micrographs of magnetic fluid with particle size of $12 \mathrm{~nm}$ at different magnetic fields perpendicular to the image plane (a) $H=0$, (b) $H=25 \mathrm{Oe}$, (c) $H=100 \mathrm{Oe}$, and (d) $H=210$ Oe.

transmittance behavior, which are useful for potential optical applications.

\section{CONCLUSIONS}

We have investigated the effect of particle size on optical transmission of light through magnetic fluid films containing magnetite nanoparticles as a function of external magnetic field and incident wavelength with different film thicknesses. For films with a particle size of $12 \mathrm{~nm}$, the transmission threshold increases from 400 to $600 \mathrm{~nm}$ monotonically as the film thickness increases from 10 to $500 \mu \mathrm{m}$. The transmission thresholds are lower for films with particle size of 7 and $9 \mathrm{~nm}$. For a given film thickness, the transmittance increases with increasing magnetic field for films with a particle size of 7 and $9 \mathrm{~nm}$, but decreases in the 12-nm film. The versatile microstructure patterns under the influence of different magnetic fields due to the balancing between van der Waals and dipole-dipole interactions of magnetic fluid nanoparticles are responsible for significant field-dependent transmittance observed.

\section{ACKNOWLEDGMENTS}

This work is supported by the National Science Council of ROC under Grants No. NSC93-2112-M-002-031 and No. NSC93-2811-M-001-015.
[1] B. Bonnemain, J. Drug Target. 6, 167 (1998).

[2] U. Hafeli, W. Schutt, J. Teller, and M. Zborowski, Scientific and Clinic Applications of Magnetic Carriers (Plenum Press, New York, 1997).

[3] W. Luo, T. Du, and J. Huang, Phys. Rev. Lett. 82, 4134
(1999).

[4] J. W. Seo, S. J. Park, and K. O. Jang, J. Appl. Phys. 85, 5956 (1999).

[5] J. E. Martin, K. M. Hill, and C. P. Tigges, Phys. Rev. E 59, 5676 (1999). 
[6] K. T. Wu and Y. D. Yao, J. Magn. Magn. Mater. 201, 186 (1999).

[7] K. T. Wu, Y. D. Yao, and T. C. Wu, Physica B 327, 319 (2003).

[8] T. Du and W. Luo, Appl. Phys. Lett. 72, 272 (1998).

[9] N. Inaba, H. Miyajima, H. Takahashi, S. Taketomi, and S. Chikazumi, IEEE Trans. Magn. 25, 3866 (1989).

[10] K. Butter, P. H. Bomans, P. M. Frederik, G. J. Vroege, and A. P. Pilipse, Nat. Mater. 2, 88 (2003).
[11] C. G. Granqvist and R. H. Buhrman, J. Appl. Phys. 47, 2200 (1976).

[12] H. E. Horng, S. Y. Yang, W. S. Tse, H. C. Yang, W. Luo, and C. Y. Hong, J. Magn. Magn. Mater. 252, 104 (2002).

[13] P. C. Scholten, in Studies of Magnetic Properties of Fine Particles and Their Relevance to Materials Science, edited by J. L. Dormann and D. Fiorani (Elsevier, Amsterdam, 1992).

[14] P. G. de Gennes and P. A. Pincus, Phys. Kondens. Mater. 11, 189 (1970). 